\title{
Acute postoperative thrombosis of an aortic valve prosthesis and embolic myocardial infarction in a coronavirus disease 2019 (COVID-19)-positive patient - an unrecognized complication
}

\author{
Nathan E. Manghat, MBChB, MRCP, FRCP, MD, FSCCT, ${ }^{\text {a }}$ Mark C. K. Hamilton, MBChB, MRCP, FRCR, ${ }^{\text {}}$ \\ Nikhil V. Joshi, MB BS, MRCP (UK), PhD, PgC Med Ed, ${ }^{\mathrm{b}}$ and \\ Hunaid A. Vohra, MB BS, MRCS, MD, FRCS (CTh), FETCS PhD, ${ }^{b}$ Bristol, United Kingdom
}

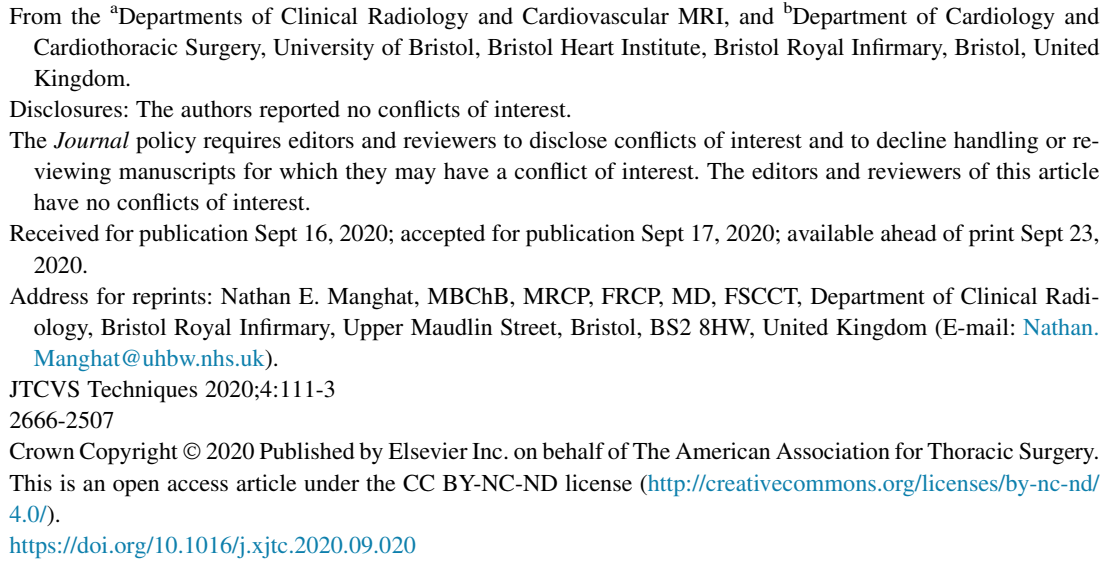

Video clip is available online.

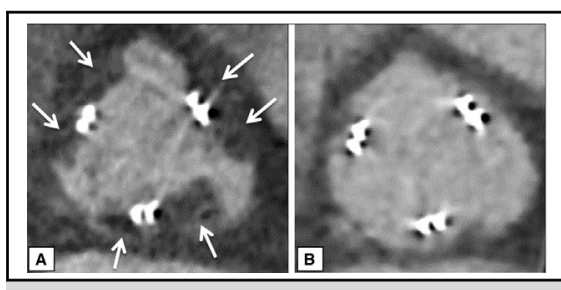

CT of the aortic root showing AVR thrombus $(A$, arrows) with resolution at 11 weeks $(B)$.

\section{CENTRAL MESSAGE \\ Clinical and radiologic vigilance is needed to diagnosis thrombosis on prosthetic AVR in COVID-19 patients. Careful thought about anticoagulation strategy and follow-up imaging is urgently required.}

See Commentaries on pages 114 and 116.
A 73-year-old female patient was readmitted with acute, sharp, central chest pain, hypotension ( $80 \mathrm{~mm} \mathrm{Hg}$ systolic), and hypoxia following a recent 25-mm diameter Carpentier-Edwards PERIMOUNT (Edwards Lifesciences, Irvine, Calif) tissue aortic valve replacement (AVR) for critical bicuspid valve stenosis and left internal mammary artery graft to the left anterior descending artery (LAD) on postoperative day 14. Medication was $75 \mathrm{mg}$ aspirin, no anticoagulants.

Electrocardiograph (ECG) findings revealed left ventricular strain pattern and 1- to 2-mm ST-segment depression in II, III, and aVF. Cardiac troponin-t at presentation was $1551 \mathrm{ng} / \mathrm{L}$, peaking at $4149 \mathrm{ng} / \mathrm{L}$. Swabs for severe acute respiratory syndrome coronavirus 2 (coronavirus disease 2019 [COVID-19]) infection were taken; preoperatively, this was negative. Findings of the coagulopathy panel were normal prothrombin time (10.9 seconds), elevated platelets $\left(488 \times 10^{9} / \mathrm{L}\right)$, fibrinogen $(6.5 \mathrm{~g} / \mathrm{L})$, and D-dimers $(5547 \mathrm{ng} / \mathrm{mL})$; preoperatively, these were all normal. ${ }^{1}$ Three sets of blood cultures were negative despite persistent low-grade pyrexia. Initial supportive oxygen and vasoconstrictor therapy resulted in clinical improvement.

ECG-gated computed tomographic (CT) angiography was performed to exclude acute aortic syndrome, graft failure, or pulmonary embolism (PE), reported by on-call radiology: left lower-lobe PE, moderate-sized hemorrhagic pericardial effusion, patent left internal mammary artery to LAD graft, no acute aortic pathology. Obesity limited transthoracic echocardiography clarity; we confirmed a pericardial effusion $1.9 \mathrm{~cm}$ in depth, no tamponade or prosthetic valve pathology with leaflets working well.

Working diagnosis was myocardial injury and secondary troponin rise due to recent cardiac surgery, PE, and large pericardial effusion. The patient had been isolated with a positive second COVID-19 swab. Transient atrial flutter and symptoms of retinal artery embolism followed shortly afterwards; serial troponin levels remained elevated (2785 ng/L). 


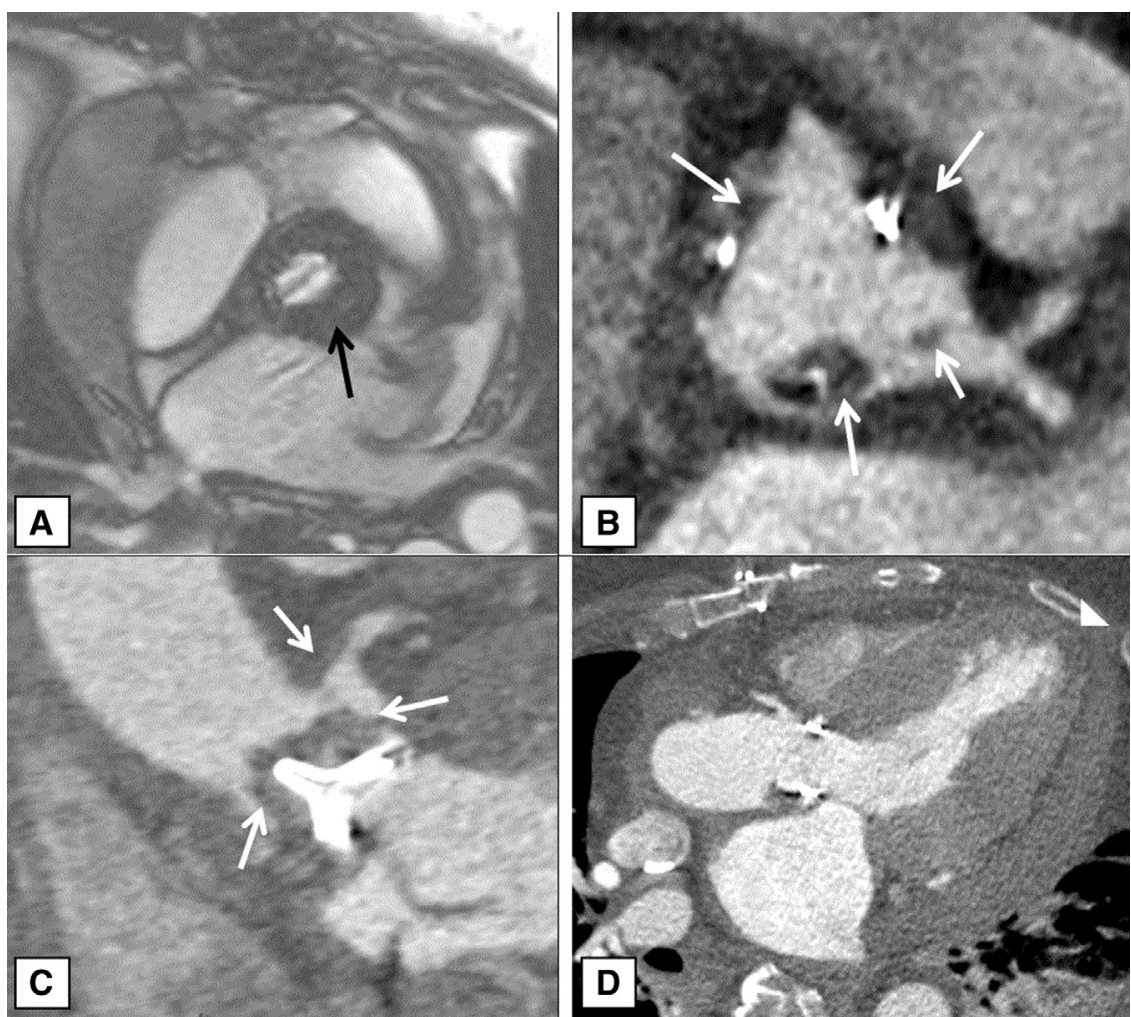

FIGURE 1. CT and MRI cross-sections. A, Oblique axial MRI-AVR low signal, metal susceptibility artifact (black arrow) cannot be separated from underlying thrombus or leaflet tissue. Oblique axial (B) and coronal (C) aortic root showing the coronary ostia and multiple foci of low attenuation soft-tissue thrombus adjacent to the AVR struts and proximal to the left main stem ostium (white arrows). D, Three-chamber long-axis ventricular systole showing apical hypokinesia with hypoperfusion (white arrowhead), no CT evidence of tamponade, and the valve leaflets were open.

Differential diagnosis was myocarditis or myocardial infarction (MI); stress perfusion cardiac magnetic resonance imaging demonstrated typical extensive acute LAD territory MI with microvascular obstruction but no ischemia, tamponade, or myocarditis. Susceptibility metallic artifact (Figure 1,A) inhibited AVR assessment.

Cardiac radiologist $\mathrm{CT}$ review also identified extensive low-attenuation, lobulated, and fragmented soft-tissue thrombus formation (Video 1) seen in the vicinity of and applied to the valve prosthesis frame (but not the leaflets) and in close proximity to the left main stem ostium (Figure 1, $B$ and $C$ ), evidence of a small acute apical MI (Figure 1, D), probable evolving perioperative hemorrhagic pericardial effusion but no tamponade or contrast extravasation and no left atrial or appendage thrombus.

Intravenous heparin (target activated partial thromboplastin time ratio 2.0-3.0) and concomitant warfarin (target international normalized ratio 2.5-3.5) was commenced 4 days after admission. The patient remained well, painfree, and was discharged 6 days later; troponin was $1257 \mathrm{ng} / \mathrm{L}$. Repeat CT was performed 11 weeks postanticoagulation commencement following a negative COVID-19 swab with the patient asymptomatic, which demonstrated complete resolution of AVR thrombosis (Figure 2, B).

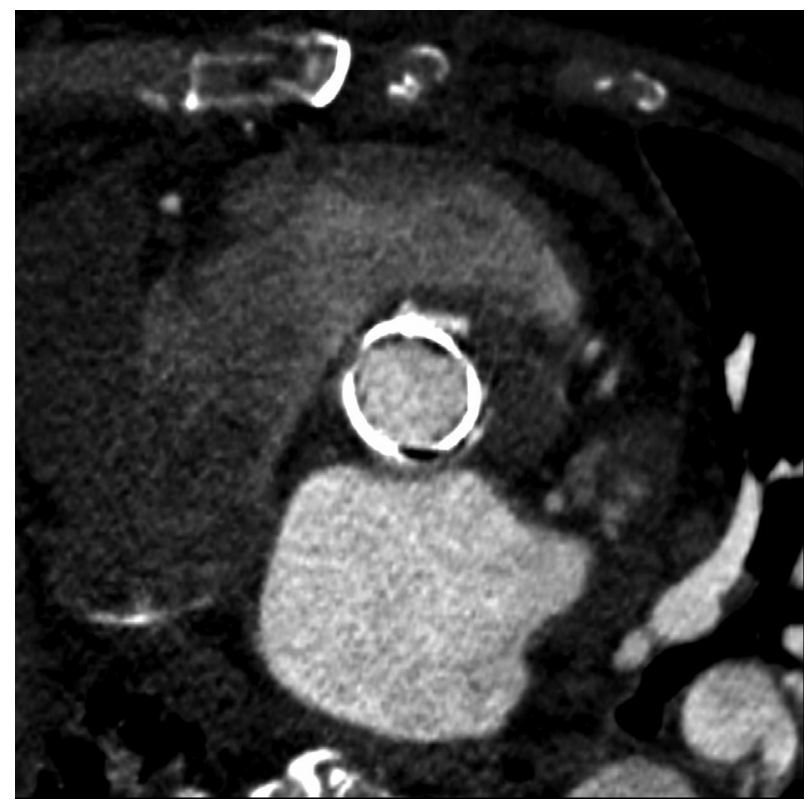

VIDEO 1. Short-axis planar reconstruction stack with 1-mm slice thickness through the biological AVR (ECG-gated, 250-millisecond phase, reconstructed using TeraRecon Intuition, Durham, NC); the lowattenuation thrombus can be clearly appreciated in relation to the AVR and in close proximity to the left main stem ostium. Video available at: https://www.jtcvs.org/article/S2666-2507(20)30534-4/fulltext. 


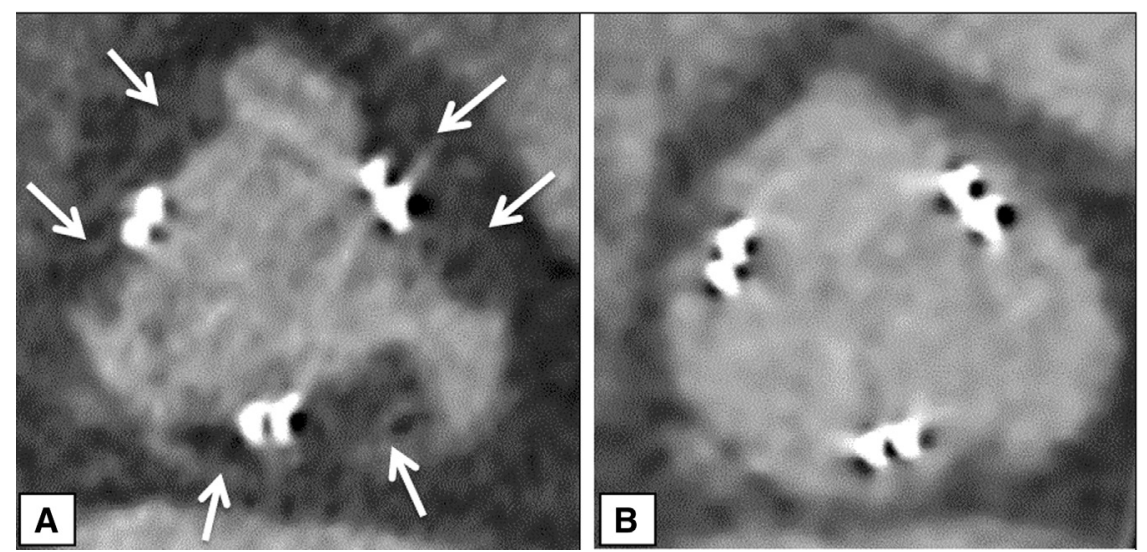

FIGURE 2. CT oblique axial cross-section through the AVR at leaflet level showing the struts (A) with extensive strut thrombus formation (white arrows) and (B) complete resolution of thrombus at 11 weeks.

This patient was negative for COVID-19 preoperatively and didn't undergo additional CT screening in accordance with recent guidelines and data. ${ }^{2}$ The pulmonary complications of COVID-19 are now well-documented, with a severe prothrombotic state association ${ }^{3}$; clinical manifestations of this tendency are poorly defined. ${ }^{4}$ Acute biological AVR thrombosis is rare ; $^{5}$ it is not usual practice to anticoagulate.

Although venous thromboembolism is now also welldocumented in patients with COVID-19, this case highlights the increased risks of systemic arterial embolism following recent cardiac procedures; the likely mechanism is a combination of the prothrombotic state and a nonendothelialized prosthetic valve frame.

This case serves as a timely reminder of the consequences of COVID-19 and the radiologic vigilance required identifying AVR thrombosis on acute cardiothoracic CT. ${ }^{6}$ The observations made warrant careful thought with respect to anticoagulation strategy; the authors recommend commencing all postoperative patients on warfarin during the COVID-19 pandemic, especially where there is clinical suspicion. ${ }^{1,3}$

Further studies are urgently needed looking at whether this phenomenon occurs more frequently by considering postimplantation (either surgically or transcatheter) ECG-gated CT in those patients with suspected COVID-19 infection. Those who develop COVID-19 postoperatively during the pandemic may require more judicious follow-up.

The patient signed a document confirming agreement that their images and data can be used for teaching, audit, and research that could benefit other patients.

\section{References}

1. The Lancet Haematology. COVID-19 coagulopathy: an evolving story. Lancet Haematol. 2020;7:e425

2. Hamilton MCK, Lyen S, Manghat NE. COntroversy in coronaViral Imaging and Diagnostics (COVID). Clin Radiol. 2020;75:557-8.

3. Connors JM, Levy JH. COVID-19 and its implications for thrombosis and anticoagulation. Blood. 2020;135:2033-40.

4. Vulliamy P, Jacob S, Davenport RA. Acute aorto-iliac and mesenteric arterial thromboses as presenting features of COVID-19. Br J Haematol. 2020;189: $1053-4$.

5. Pradegan N, Basso C, Della Barbera M, Thiene G, Tarantini G, Gerosa G et al. Cardiac arrest due to acute massive aortic root thrombosis after pericardial bioprosthetic aortic valve replacement. Cardiovasc Pathol. 2019;41:8-10.

6. Manghat NE, Rachapalli V, Van Lingen R, Veitch AM, Roobottom CA, MorganHughes GJ. Imaging the heart valves using ECG-gated 64-detector row cardiac CT. Br J Radiol. 2008;81:275-90. 